\title{
Exploring colistin pharmacodynamics against Klebsiella pneumoniae: a need to revise current susceptibility breakpoints
}

\author{
Marilena Tsala ${ }^{1}$, Sophia Vourli ${ }^{1}$, Panagiota-Christina Georgiou ${ }^{1}$, Spyros Pournaras ${ }^{1,2}$, Athanasios Tsakris $^{2}$, \\ George L. Daikos ${ }^{3}$, Johan W. Mouton ${ }^{4}$ and Joseph Meletiadis ${ }^{1,4 *}$
}

${ }^{1}$ Clinical Microbiology Laboratory, Attikon University Hospital, Medical School, National and Kapodistrian University of Athens, Athens, Greece; ${ }^{2}$ Department of Microbiology, Medical School, National and Kapodistrian University of Athens, Athens, Greece; ${ }^{3}$ First Department of Propaedeutic Medicine, Laikon Hospital, Medical School, National and Kapodistrian University of Athens, Athens, Greece; ${ }^{4}$ Department of Medical Microbiology and Infectious Diseases, Erasmus Medical Center, Rotterdam, The Netherlands

*Corresponding author. 1 Rimini St, Haidari 124 62, Athens, Greece. Tel: +30-210-583-1909; Fax: +30-210-532-6421; E-mail:jmeletiadis@med.uoa.gr

Received 24 August 2017; returned 9 October 2017; revised 7 December 2017; accepted 13 December 2017

Objectives: Because the pharmacokinetic/pharmacodynamic (PK/PD) characteristics of colistin against Enterobacteriaceae are not well explored, we studied the activity of colistin against $K$. pneumoniae in an in vitro PK/PD model simulating different dosing regimens.

\begin{abstract}
Methods: Three clinical isolates of $K$. pneumoniae with MICs of $0.5,1$ and $4 \mathrm{mg} / \mathrm{L}$ were tested in an in vitro PK/PD model following a dose-fractionation design over a period of $24 \mathrm{~h}$. A high and low inoculum of $10^{7}$ and $10^{4} \mathrm{cfu} / \mathrm{mL}$ with and without a heteroresistant subpopulation, respectively, were used. PK/PD indices associated with colistin activity were explored and Monte Carlo analysis was performed in order to determine the PTA for achieving a bactericidal effect ( 2 log kill).
\end{abstract}

Results: The fAUC/MIC $\left(R^{2}=0.64-0.68\right)$ followed by $f C_{\max } / \mathrm{MIC}\left(R^{2}=0.55-0.63\right)$ best described colistin's $24 \mathrm{~h}$ $\log _{10} \mathrm{cfu} / \mathrm{mL}$ reduction for both low and high inocula. Dosing regimens with $f C_{\max } / \mathrm{MIC} \geq 6$ were always associated with a bactericidal effect $(P=0.0025)$. However, at clinically achievable concentrations, usually below $f C_{\max } / \mathrm{MIC}=6$, an $f A U C / M I C \geq 25$ was more predictive of a bactericidal effect. Using a dosing regimen of $9 \mathrm{MU} /$ day, the PTA for this pharmacodynamic target was $100 \%, 5 \%-70 \%$ and $0 \%$, for isolates with MICs of $\leq 0.5,1$ and $\geq 2 \mathrm{mg} / \mathrm{L}$, respectively. Dosing regimens that aim for a trough level of $1 \mathrm{mg} / \mathrm{L}$ achieve coverage of strains up to $0.5 \mathrm{mg} / \mathrm{L}$ (target trough $/ \mathrm{MIC}=2 \mathrm{mg} / \mathrm{L}$ ).

Conclusions: Characterization of the pharmacodynamics of colistin against Enterobacteriaceae in an in vitro model of infection indicates that a revision of current susceptibility breakpoints is needed. Therapeutic drug monitoring of colistin to achieve pharmacodynamic targets in individual patients is highly recommended.

\section{Introduction}

The emergence of MDR Gram-negative bacteria, including carbapenemase-producing Klebsiella pneumoniae (CP-Kp), isolates has led to the revival of the use of old antibiotics such as colistin. ${ }^{1,2}$ Understanding the pharmacodynamics of colistin against CP-Kp is important in order to optimize antimicrobial therapy against these infections. Although there are several preclinical pharmacokinetic/ pharmacodynamic (PK/PD) studies of Pseudomonas aeruginosa and Acinetobacter baumannii in in vitro pharmacokinetic and animal models, ${ }^{3}$ the PK/PD characteristics of colistin against Enterobacteriaceae have not been extensively explored. Here we focused on the pharmacodynamics of colistin against K. pneumoniae, and used an in vitro PK/PD model to simulate different colistin exposures against low and high inocula. We subsequently calculated the PTA for isolates with different MICs to evaluate the susceptibility breakpoint of colistin.

\section{Materials and methods}

\section{Isolates, drug and medium}

Three clinical isolates of $K$. pneumoniae with colistin CLSI MICs of $0.5 \mathrm{mg} / \mathrm{L}$ (WT TZAN59), $1 \mathrm{mg} / \mathrm{L}$ (carbapenemase-producing KPC1433) and $4 \mathrm{mg} / \mathrm{L}$ (verified in quadruplicate experiments) were used. The isolates were stored at $-70^{\circ} \mathrm{C}$ and revived after subculturing on MacConkey agar plates at $37^{\circ} \mathrm{C}$ for $18-24 \mathrm{~h}$, and final concentrations of $10^{7}$ and $10^{4} \mathrm{cfu} / \mathrm{mL}$, verified by quantitative cultures, were used as starting inocula. Colistin sulfate (Sigma Aldrich, Athens, Greece) and cation-adjusted Mueller-Hinton broth 
were used according to CLSI instructions. ${ }^{4}$ The two inocula were chosen based on preliminary experiments to assess the presence of heteroresistant subpopulations. Briefly, $100 \mu \mathrm{L}$ of 10 -fold increasing inocula $\left(10^{3}-10^{7} \mathrm{cfu} / \mathrm{mL}\right)$ of TZAN59 and KPC1433 isolates were cultured on Mueller-Hinton agar containing 2-fold concentrations of colistin ranging from 2 to $64 \mathrm{mg} / \mathrm{L}$ and the number of colonies grown after $24 \mathrm{~h}$ were counted and MICs determined. No resistant subpopulations were found at inocula up to $10^{4} \mathrm{cfu} / \mathrm{mL}$ whereas $1-13 \mathrm{cfu}$ were grown on Mueller-Hinton agar containing $8-32 \mathrm{mg} / \mathrm{L}$ with the $10^{7} \mathrm{cfu} / \mathrm{mL}$ inoculum.

\section{In vitro PK/PD model}

A previously developed closed diffusion/dialysis in vitro pharmacokinetic model was used in the present study in order to simulate the pharmacokinetics of colistin in humans and to study its antibacterial effect. ${ }^{5,6} \mathrm{~A}$ dosefractionation design was followed with nine dosing regimens of colistin targeting $f C_{\max } 9,3$ and $1.5 \mathrm{mg} / \mathrm{L}$ administered every 8,12 and $24 \mathrm{~h}$ for $24 \mathrm{~h}$. High drug exposures were included in order to better describe the exposure-effect relationships with effects ranging from low to high. Colistin was added to both compartments of the in vitro model in order to reach a peak concentration within $1.5 \mathrm{~h}$, simulating exposures achieved with a loading dose followed by maintenance doses. The activity of additional dosing regimens with increasing $f C_{\max }$ and longer half-lives was also assessed. Drug concentrations in the internal compartment were determined with a microbiological diffusion assay using $10^{6} \mathrm{cfu} / \mathrm{mL}$ Escherichia coli ATCC 25922 impregnated in antibiotic medium 10 agar in Mueller-Hinton broth (Difco, Athens, Greece) (concentration range $0.25-16 \mathrm{mg} / \mathrm{L}$ with $r^{2}=0.98$ and inter-day variation $<7 \%)^{7}$

\section{PK/PD analysis}

The PK/PD relationships were analysed by non-linear regression using the $E_{\max }$ model described by the equation $E=E_{\max } \times\left(E I / E_{50}\right)^{m} /[1+(E I /$ $\left.E I_{50}\right)^{m}$ ], where $E$ is the bacterial load at the end of experiment (dependent variable) in $\log _{10} \mathrm{cfu} / \mathrm{mL}$, EI is the PK/PD index $f C_{\text {max }} / \mathrm{MIC}$, $f A U C / M I C$ or $\% f T_{>M I C}$ (independent variable), $E_{\max }$ is the maximum bacterial load in $\log _{10} \mathrm{cfu} / \mathrm{mL}$ observed in the drug-free control group, $\mathrm{EI}_{50}$ is the EI corresponding to $50 \%$ of $E_{\max }$ and $m$ is the slope of the concentration-effect curves (Hill coefficient) (GraphPad Prism 4.03, San Diego, CA). Colistin exposures associated with a bacteriostatic effect (i.e. no change compared with the initial inoculum after $24 \mathrm{~h}$ ) and a 2 log kill effect (i.e. $2 \log _{10} \mathrm{cfu} / \mathrm{mL}$ reduction from initial inoculum) were calculated. The 2 log kill effect in the present model was previously found to be associated with 1 log kill in a thigh infection murine model. ${ }^{8}$ In order to capture pharmacodynamic effects for the entire $24 \mathrm{~h}$ period, a similar analysis was performed using the area under the $24 \mathrm{~h}$ time-kill curve (AUTKC) normalized to span from 100\% (drug-free control) to $0 \%\left(\log _{10} L O D \times 24\right)$. Bactericidal effects were analysed with classification and regression tree (CART) analysis, using as a response the presence of bactericidal activity as the nominal value and all three PK/PD indices as continuous variables. The results of the CART analysis were assessed statistically with Fisher's exact test.

\section{Monte Carlo simulations and analysis}

The probability of attaining a 2 log kill effect against $K$. pneumoniae isolates was calculated by applying Monte Carlo simulations of 5000 patients with normal renal function (mean $\mathrm{CL}_{\mathrm{CR}}$ 85-105) treated with either $9 \mathrm{MU}$ q24h, 4.5 MU q12h or $3 \mathrm{MU}$ q8h. These dosing regimens were previously found to result in $t C_{\max } \pm S D 5.83 \pm 0.87,2.98 \pm 0.27$ and $3.34 \pm 0.35 \mathrm{mg} / \mathrm{L}$; total $t C_{\min } \pm S D 2.60 \pm 1.12,2.01 \pm 0.47$ and $1.63 \pm 0.23 \mathrm{mg} / \mathrm{L}$; and $t A U C_{0-24} \pm S D$ $72.93 \pm 38.57,60.71 \pm 12.0$ and $50.18 \pm 10.74 \mathrm{mg} \cdot \mathrm{h} / \mathrm{L}$, respectively. ${ }^{9}$ The Monte Carlo simulation was performed using the normal random number generator function of Excel (MS Office 2007) and the corresponding $f C_{\max }$ and FAUC were calculated based on the $40 \%$ of unbound fraction of colistin in human serum. ${ }^{8}$ The PK/PD PTA associated with a 2 log kill effect was calculated for each MIC and dosing regimen. The PTA at the epidemiological cut-off (ECOFF) of colistin for K. pneumoniae ( $2 \mathrm{mg} / \mathrm{L}$ ) was also estimated.

The cumulative fraction of response $(C F R)^{10}$ was calculated for K. pneumoniae with the WT MIC distribution as presented on the EUCAST web site with the following frequencies: $\leq 1 \%, 20 \%, 55 \%, 18 \%$ and $\leq 2 \%$ for MICs $\leq 0.125,0.25,0.5,1$ and $\geq 2 \mathrm{mg} / \mathrm{L}$, respectively. In addition, the CFR was also calculated for a hypothetical collection of isolates with MICs shifted by one, two and three 2-fold dilutions higher than the EUCAST MIC distribution described above, resulting in three MIC distributions with modal MICs of 1,2 and $4 \mathrm{mg} / \mathrm{L}$ and resistance rates (isolates with MIC $>2 \mathrm{mg} / \mathrm{L}$ ) of $3 \%, 20 \%$ and $73 \%$, respectively. Finally, the trough levels required with each dosing regimen in order to achieve a bactericidal effect for isolates with increasing MICs were calculated taking into account the $40 \%$ unbound fraction, ${ }^{8}$ a $1 / 30 \mathrm{AUC} / \mathrm{C}_{\min }$ ratio $^{9}$ and a $t_{1 / 2}$ of $12 \mathrm{~h}^{9,11}$

\section{Results}

\section{In vitro pharmacodynamics}

For the higher inoculum, after a concentration-dependent decrease in $\log _{10} \mathrm{cfu} / \mathrm{mL}$ within the first $2 \mathrm{~h}$, regrowth was observed for some dosing regimens, in particular for the strain KPC1433 (Figure 1). A 2 log kill effect was observed for the q8h dosing regimen with $f C_{\text {max }} \geq 1 \mathrm{mg} / \mathrm{L}$. For regimens with longer dosing intervals, a bactericidal effect was observed for the $q 12 \mathrm{~h}$ dosing regimens with $f C_{\text {max }} \geq 4.5$ for $\mathrm{KPC} 1433$, and $f C_{\text {max }} \geq 0.75 \mathrm{mg} / \mathrm{L}$ for TZAN59 and for the $\mathrm{q} 24 \mathrm{~h}$ dosing regimens with $f C_{\max } \geq 6 \mathrm{mg} / \mathrm{L}$ for $K P C 1433$ and $f C_{\text {max }} \geq 3 \mathrm{mg} / \mathrm{L}$ for TZAN59. Resistant subpopulations were found at both $t=0 \mathrm{~h}$ and $t=24 \mathrm{~h}$. The MICs of these populations were higher $(32 \mathrm{mg} / \mathrm{L}$ ) than the MICs of the initial isolates.

For the lower inoculum, a decrease in $\log _{10} \mathrm{cfu} / \mathrm{mL}$ was also observed within $2 \mathrm{~h}$. However, this was not concentration dependent since all dosing regimens reduced bacterial load to below the LOD (data not shown). No resistant subpopulations were found at $t=0 \mathrm{~h}$ and $t=24 \mathrm{~h}$. The MICs of colonies grown on drug-free media at $24 \mathrm{~h}$ were similar to the MICs of initial isolates, whereas the MICs of colonies grown on colistin-containing media were higher.

\section{In vitro PK/PD relationships}

The PK/PD relationships for the $24 \mathrm{~h} \log _{10} \mathrm{cfu} / \mathrm{mL}$ and the $24 \mathrm{~h}$ AUTKC are shown in Figure 2. For the highest inoculum of $10^{7} \mathrm{cfu} / \mathrm{mL}$ and the $24 \mathrm{~h} \log _{10} \mathrm{cfu} / \mathrm{mL}$ change, the $R^{2}$ for all three PK/PD indices were in the range 0.55-0.68, with the fAUC/MIC showing the highest $R^{2}$. Large variability between the two strains was observed for intermediate exposures (fAUC/MIC 10-30 and $\mathrm{fC}_{\text {max }} / \mathrm{MIC} 1$-4) with $24 \mathrm{~h} \log _{10} \mathrm{cfu} / \mathrm{mL}$ change varying from $2 \log _{10}$ growth to $6 \log _{10}$ kill. This variability was minimized when the $24 \mathrm{~h}$ AUTKC was used to express outcome as a function of fAUC/MIC, giving a slightly higher $R^{2}(0.90)$ compared with $f C_{\max } / \mathrm{MIC}$ $\left(R^{2}=0.83\right)$ and $\% f T_{>M I C}\left(R^{2}=0.79\right)$. The largest variability was again observed at intermediate drug exposures with effects varying from $20 \%$ to $80 \%$ of $E_{\text {max }}$.

Similar results were found with the lower inoculum with both fAUC/MIC $\left(R^{2}=0.64\right.$ for $24 \mathrm{~h} \log _{10} \mathrm{cfu} / \mathrm{mL}$ change and $R^{2}=0.76$ for $24 \mathrm{~h}$ AUTKC) and $f C_{\text {max }} / \mathrm{MIC}\left(R^{2}=0.63\right.$ for $24 \mathrm{~h} \log _{10} \mathrm{cfu} / \mathrm{mL}$ change and $R^{2}=0.62$ for $24 \mathrm{~h}$ AUTKC), which clearly offer a better description than than $\% f T_{>\text {MIC }}\left(R^{2}=0.51\right.$ for $24 \mathrm{~h} \log _{10} \mathrm{cfu} / \mathrm{mL}$ change and $R^{2}=0.44$ for $24 \mathrm{~h}$ AUTKC) (data not shown). 

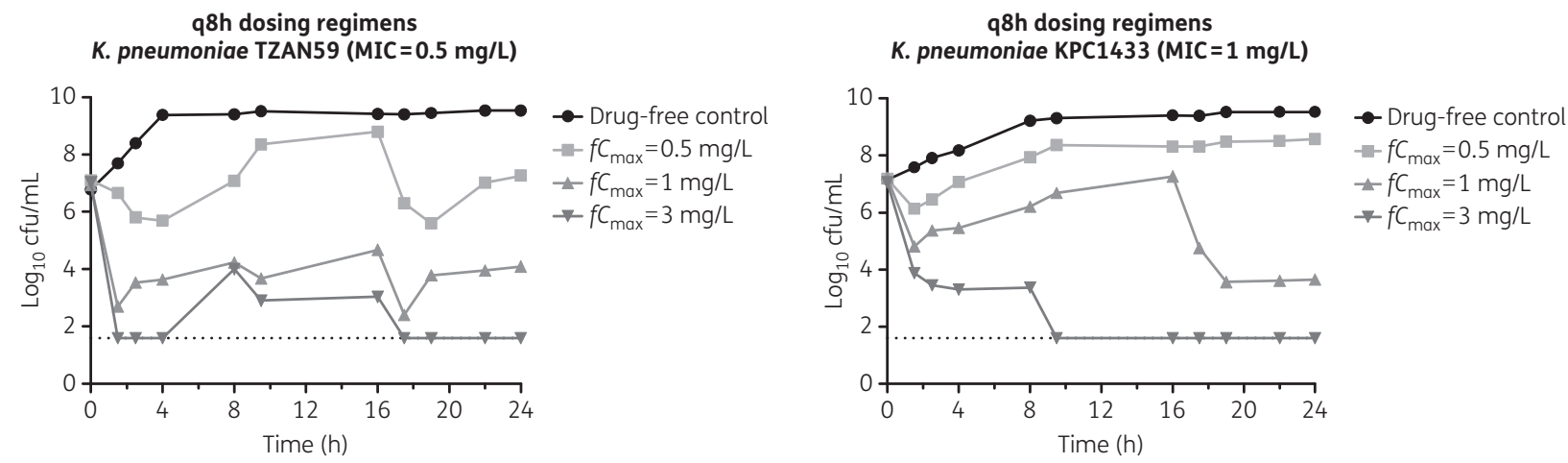

$\mathrm{q} 12 \mathrm{~h}$ dosing regimens K. pneumoniae TZAN59 (MIC $=0.5 \mathrm{mg} / \mathrm{L}$ )

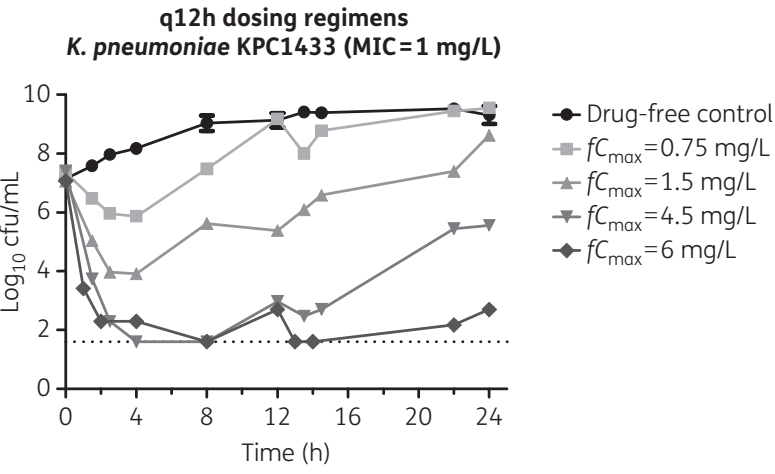
$\rightarrow$ Drug-free control
$\rightarrow f C_{\max }=0.75 \mathrm{mg} / \mathrm{L}$
$\rightarrow f C_{\max }=1.5 \mathrm{mg} / \mathrm{L}$
$\rightarrow f C_{\max }=4.5 \mathrm{mg} / \mathrm{L}$

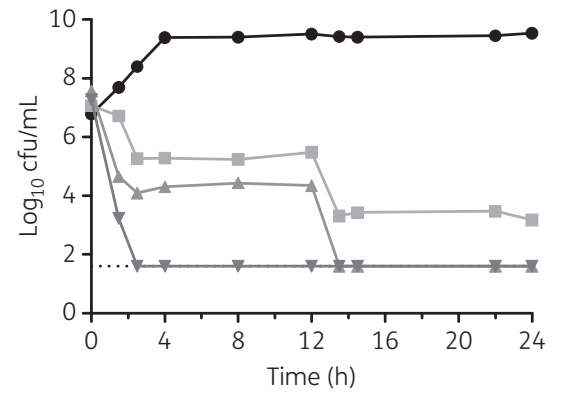

q24h dosing regimens
K. pneumoniae TZAN59 (MIC $=0.5 \mathrm{mg} / \mathrm{L})$

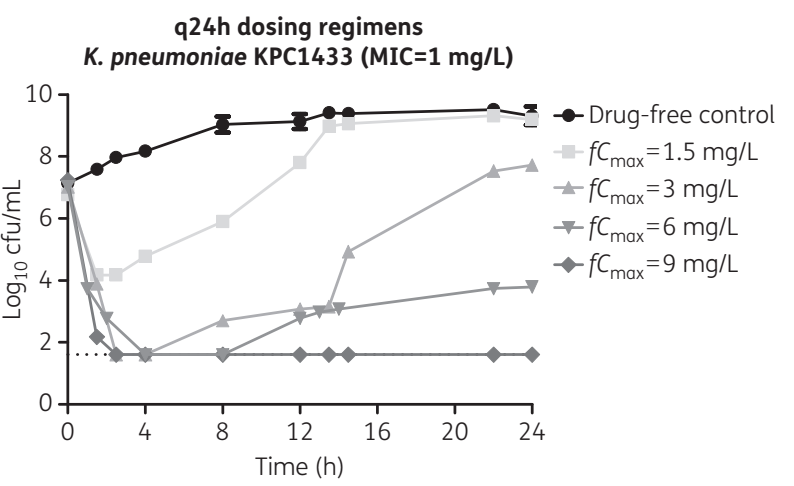

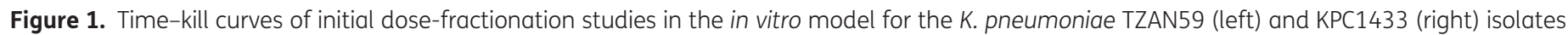

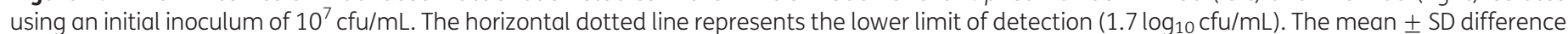
between measured and target peak concentrations was $13 \pm 5 \%$.

Given the relatively small $R^{2}$ of exposure-effect relationships, CART analysis was performed in order to determine an EI strongly associated with a 2 log kill effect. CART analysis showed that both $f C_{\text {max }} / \mathrm{MIC}$ and $f A U C / M I C$ were associated with 2 log kill for the high inoculum. Dosing regimens with an $f C_{\max } / \mathrm{MIC}$ ratio of $\geq 6$ and an fAUC/MIC ratio of $\geq 30$ resulted in 100\% (10/10) 2 log kill, whereas this was only $38 \%$ (6/16) with lower $f C_{\text {max }} /$ MIC and $f A U C / M I C$ ratios $(P=0.0014)$. Likewise, for the low inoculum, an $f C_{\max } / \mathrm{MIC} \geq 6$ $(P=0.0025)$ and an $f A U C / M I C$ ratio of $\geq 25(P=0.013)$ was associated with $2 \log$ kill.

In order to elucidate the impact of $f C_{\max }$ and $f A \cup C$ on colistin pharmacodynamics separately, dosing regimens with different $f C_{\text {max }}$ and $f A U C s$ were simulated in additional experiments in the in vitro model targeting increasing PK/PD index values. A $1 \mathrm{~h}$ infusion and a $10 \mathrm{~h}$ infusion were applied to achieve $f C_{\max } 1-6 \mathrm{mg} / \mathrm{L}$ and
fAUCs varying from 7.5 to $32 \mathrm{mg} \cdot \mathrm{h} / \mathrm{L}$ against TZAN59 and KPC1433. Confirming the results in the initial experiments, a high $f C_{\max } / \mathrm{MIC}$ ratio appeared to have a beneficial effect. A bactericidal effect was found for dosing regimens with $f C_{\max } / \mathrm{MIC} \geq 6$ against TZAN59 even with an FAUC/MIC as low as 15 (Figure 3). By contrast, for dosing regimens with low $f C_{\max } / \mathrm{MIC}$ ratios between 1 and 6 , bactericidal effects were less apparent and observed only at high fAUC/MIC ratios between 30.6 and 61 (Figure $4 a$ and b). In another set of experiments simulating dosing regimens targeting $f C_{\text {max }} /$ MIC (fAUC/MIC) ratios of 3 (32), 6 (21), 6 (62) and 12 (42), complete kill was found for all dosing regimens with either $f C_{\max } / \mathrm{MIC} \geq 6$ or $f A \cup C / M I C \geq 32$ (Figure $4 \mathrm{C}$ and $\mathrm{d}$ ). The simulated dosing regimens with $f C_{\max } 32 \mathrm{mg} / \mathrm{L}$ q $24 \mathrm{~h}$ and $t^{1} / 2=2 \mathrm{~h}$ (obtained $f C_{\max } / \mathrm{MIC} 6.1$ and $\left.f A U C / M I C 23.5\right)$ and $f C_{\max } 16 \mathrm{mg} / \mathrm{L} \mathrm{q} 12 \mathrm{~h}$ and $t^{1} / 2=12 \mathrm{~h}$ (obtained $f C_{\max } / M I C 3.8$ and $f A U C / M I C$ 40.6) were 

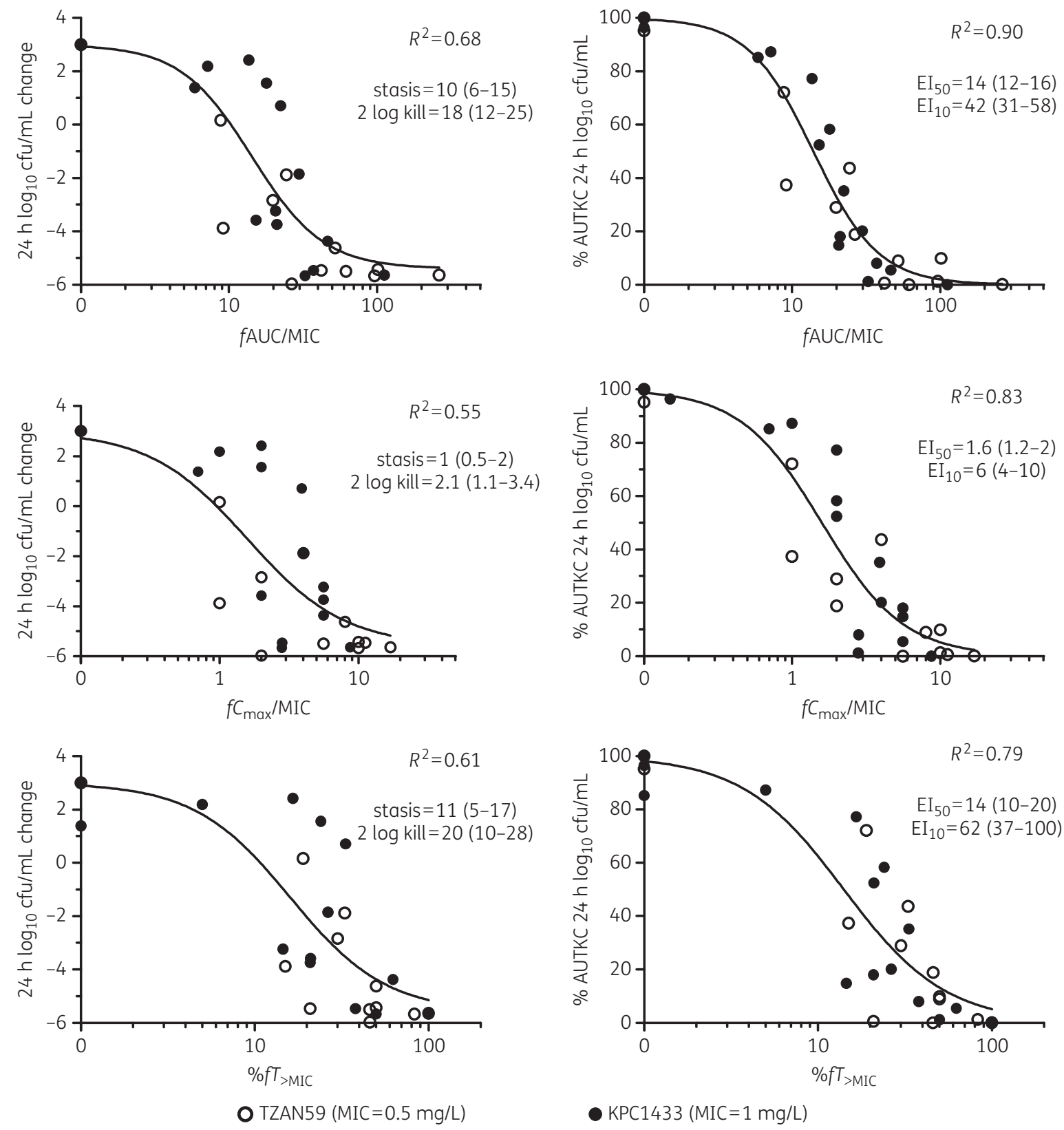

- KPC1433 (MIC=1 mg/L)

Figure 2. The in vitro PK/PD relationships fAUC/MIC (top), $f C_{\text {max }} / M I C$ (middle) and \% $f T_{>M I C}$ (bottom) of colistin using the $24 \mathrm{~h}$ log $10 \mathrm{cfu} / \mathrm{mL}$ change compared with the initial inoculum (left graphs) and the normalized percentage area under the $24 \mathrm{~h}$ time-kill curve (AUTKC) compared with the AUTKC of drug-free controls (right graphs) using an initial inoculum of $10^{7} \mathrm{cfu} / \mathrm{mL}$.

effective in achieving a 2 log kill effect of the low-level resistant K. pneumoniae isolate. Thus, all experiments indicate that both $f C_{\max } / \mathrm{MIC}$ and $\mathrm{fAUC} / \mathrm{MIC}$ ratios are important targets for efficacy.

\section{Target attainment}

Using the results above, the pharmacodynamic targets $f C_{\text {max }} / M I C=6$ and $f A U C / M I C=25$ were chosen in order to estimate the PTA since both targets were associated with 2 log kill activity against $K$. pneumoniae. The PTAs are shown in Figure 5 for the three most often used clinical dosing regimens of $9 \mathrm{MU}$ q24h,
4.5 $\mathrm{MU}$ q12h and $3 \mathrm{MU}$ q8h. An $f C_{\max } / \mathrm{MIC}>6$ was attained for isolates with MIC $\leq 0.25 \mathrm{mg} / \mathrm{L}$ for most $(>90 \%)$ patients treated with $9 \mathrm{MU}$ q24h and for isolates with an MIC $\leq 0.125 \mathrm{mg} / \mathrm{L}$ for most patients treated with $4.5 \mathrm{MU}$ q12h and $3 \mathrm{MU}$ q8h (similar results were found with the $3 \mathrm{log}$ kill). However, fAUC/MIC $>25$ was attained in $100 \%, 5 \%-70 \%$ and $0 \%$ for isolates with MICs of $\leq 0.5$, 1 and $\geq 2 \mathrm{mg} / \mathrm{L}$, respectively, with all three dosing regimens (Figure 5). Thus, the PTA was 0 for the current breakpoint of $2 \mathrm{mg} / \mathrm{L}$. The CFRs for $K$. pneumoniae isolates following the EUCAST MIC distribution with a modal MIC of $0.5 \mathrm{mg} / \mathrm{L}$ and a resistance rate (isolates with MIC $>2 \mathrm{mg} / \mathrm{L}$ ) of $2 \%$ were comparable for the three 

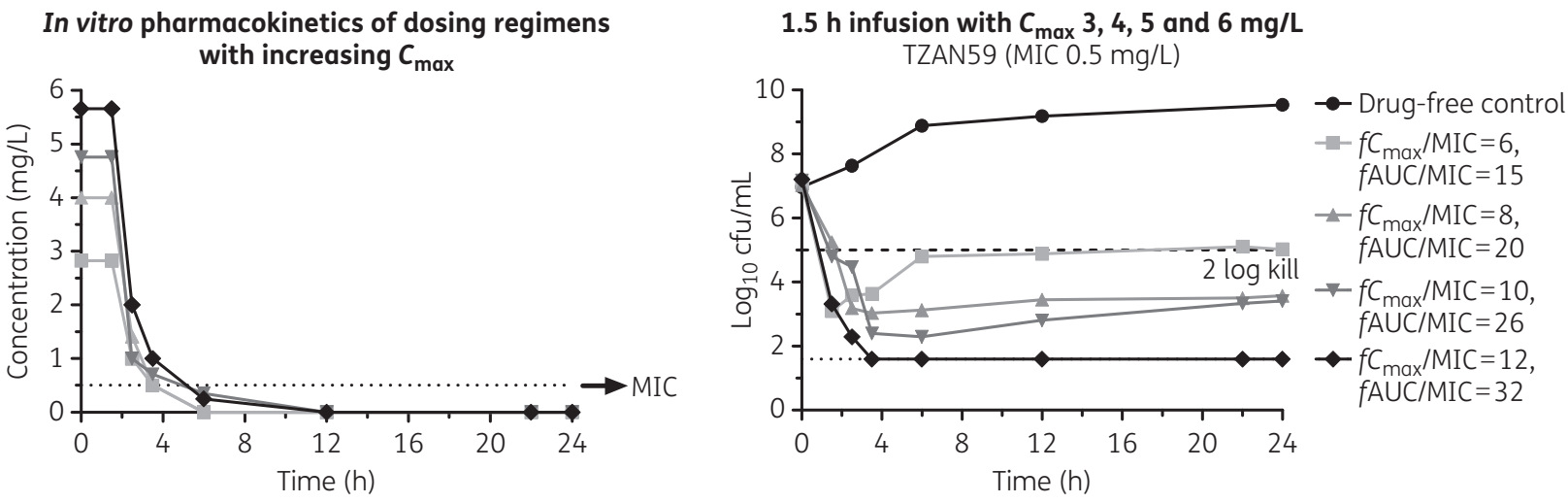

Figure 3. In vitro pharmacodynamics of different dosing regimens with increasing $f C_{\max }$ against the WT (TZAN59) K. pneumoniae isolate. Left graph: concentration-time profiles of dosing regimens used. Right graph: killing curves of four dosing regimens.
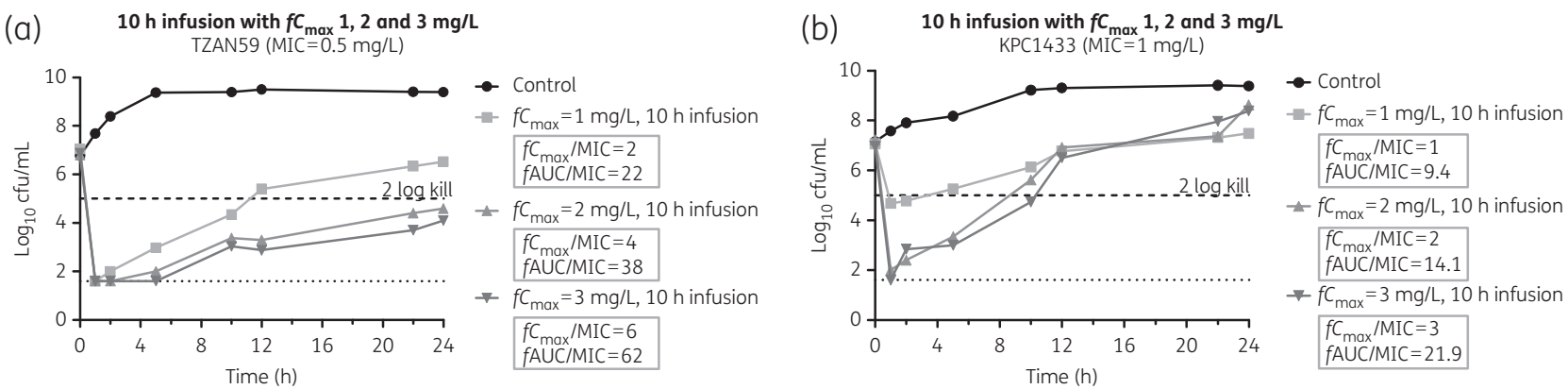

$1.5 \mathrm{~h}$ infusion with $f C_{\max } 6 \mathrm{mg} / \mathrm{L}$ versus
$10 \mathrm{~h}$ infusion with $f C_{\text {max }} 3 \mathrm{mg} / \mathrm{L}$

(d) $\quad 1.5 \mathrm{~h}$ infusion with $f C_{\text {max }} 6 \mathrm{mg} / \mathrm{L}$ versus TZAN59 (MIC $=0.5 \mathrm{mg} / \mathrm{L}$ ) $10 \mathrm{~h}$ infusion with $f C_{\max } 3 \mathrm{mg} / \mathrm{L}$

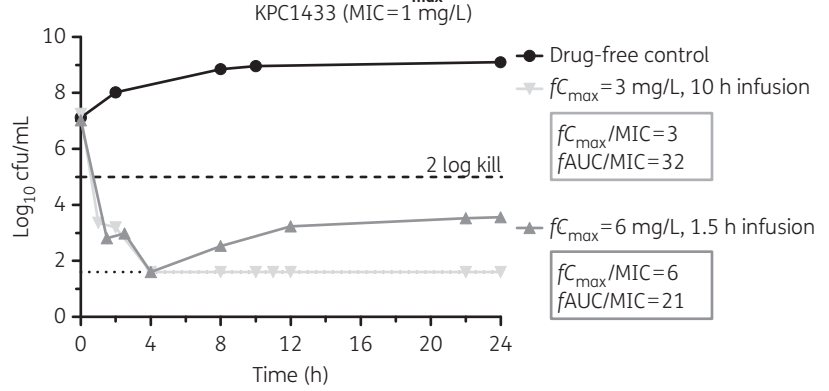

Figure 4. In vitro pharmacodynamics of different dosing regimens with 1.5 and $10 \mathrm{~h}$ infusion and increasing $f C_{\max }$ against (a, c) the carbapenemaseproducing KPC1433 K. pneumoniae isolate, and (b, d) a WT (TZAN59) K. pneumoniae isolate.

dosing regimens $(76 \%-83 \%)$ with the highest CFR found for the $3 \mathrm{MU}$ q8h (Figure 6a). For MIC distributions with higher modal MICs and resistance rates of $1 \mathrm{mg} / \mathrm{L}$ and $3 \%$ (Figure $6 \mathrm{~b}$ ), $2 \mathrm{mg} / \mathrm{L}$ and $20 \%$ (Figure $6 \mathrm{c}$ ), and $4 \mathrm{mg} / \mathrm{L}$ and $73 \%$ (Figure $6 \mathrm{~d}$ ), the CFR was $82 \%, 39 \%, 8 \%$ and $0 \%$ for 3 MU q8h, respectively.

The trough levels required to attain a 2 log kill effect for isolates with increasing MICs are shown in Figure 7. In order to attain the PK/PD target FAUC/MIC $>25$, the estimated target trough/MIC ratio was 2 , indicating that isolates with an MIC up to $1 \mathrm{mg} / \mathrm{L}$ could be covered with a non-toxic dosing regimen (trough levels $<3.33 \mathrm{mg} / \mathrm{L}$ ). ${ }^{12}$ This could just be achieved with $3 \mathrm{MU}$ q8h and 4.5 MU q24h. However, $1 \mathrm{mg} / \mathrm{L}$ is below the breakpoint of $2 \mathrm{mg} / \mathrm{L}$ and colistin therapy is therefore marginal at best. Most patients treated with $9 \mathrm{MU}$ q24h could achieve those levels but the large interindividual variation and the fact that one-quarter of the patients would have toxic trough levels indicates the necessity for therapeutic drug monitoring (TDM).

\section{Discussion}

In vitro PK/PD modelling of colistin showed that both $f C_{\max } / \mathrm{MIC}$ and $F A U C / M I C$ described colistin's activity against low and high inocula of K. pneumoniae. At low inoculum, regrowth was associated with adaptive resistance since recovered isolates grown in drug-free media had low MICs. In contrast, for the high inoculum, regrowth was associated with heteroresistance since recovered subpopulations of isolates had high MICs. Dosing regimens with $f C_{\text {max }} / \mathrm{MIC}>6$ were associated with $a>2$ log kill effect independently of $f A U C$. However, for dosing regimens with lower $f C_{\max } / \mathrm{MIC}$ ratios, an $\mathrm{FAUC/MIC}>25$ appeared to be more predictive of $>2 \mathrm{log}$ 
CMS 9 MU q24h for K. pneumoniae

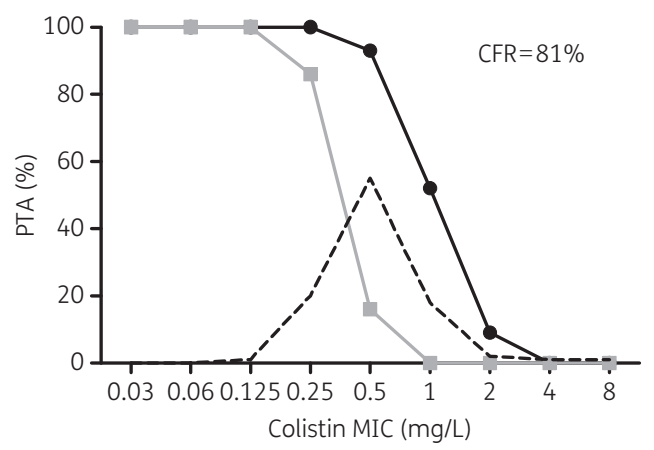

CMS 3 MU q8h for K. pneumoniae

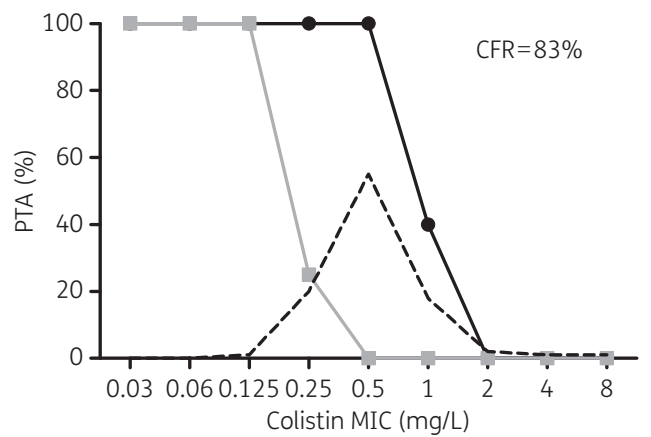

CMS 4.5 MU q12h for K. pneumoniae

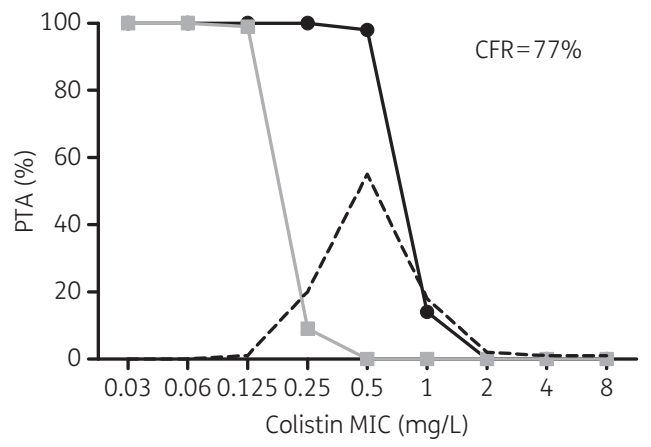

fAUC/MIC $=25$

$\rightarrow-f C_{\max } / M I C=6$

-.. EUCAST MIC

Figure 5. Probability of target attainment for K. pneumoniae isolates with increasing MICs and three clinical dosing regimens of colistin administered in ICU patients with normal renal function ${ }^{11}$ for two PD targets: $f A U C / M I C=25$ (black line) and $f C_{\text {max }} / \mathrm{MIC}=6$ (grey line). The cumulative fraction of response (CFR) is shown for a collection of isolates with the EUCAST MIC distribution with a modal MIC of $0.5 \mathrm{mg} / \mathrm{L}$ (broken line).

kill activity. Because conventional clinical dosing regimens usually result in low serum $f C_{\text {max }} /$ MIC ratios, the apparently determining PK/PD index in patients is the FAUC/MIC. Although the PTA for an fAUC/MIC $>25$ was $100 \%$ for isolates with an MIC $\leq 0.5 \mathrm{mg} / \mathrm{L}$, it decreased to $0 \%$ at $2 \mathrm{mg} / \mathrm{L}$. The latter is the ECOFF for K. pneumoniae, indicating that colistin is likely not optimally effective as monotherapy. The clinical breakpoint at present, however, is $2 \mathrm{mg} / \mathrm{L} .{ }^{13}$ The CFR for WT isolates was $82 \%$, and dropped significantly when shifts in distributions were simulated.

The PK/PD properties of colistin against Enterobacteriaceae have not been previously explored in detail. Preclinical studies on using colistin in animals against $P$. aeruginosa and $A$. baumannii showed that $F A U C / M I C$ best correlated with bacterial killing. The FAUC/MIC required for a $1 \log _{10}$ kill in animal thigh and lung infections models was 12.2-22.8 for $P$. aeruginosa and 7-42 for A. baumannii. ${ }^{3}$ This is in agreement with the fAUC/MIC found in the present study against $K$. pneumoniae. In a previous animal pneumonia model using K. pneumoniae, a colistin AUC/MIC of 158.5 was associated with $68 \%$ mortality. ${ }^{14}$ Since colistin protein binding in mouse serum is $90 \%-92 \%,{ }^{15}$ the fAUC/MIC should be $12-16$, which is within the $F A U C / M I C$ range found in the present study.

Although fAUC/MIC accurately describes colistin activity, $f C_{\text {max }} / \mathrm{MIC}$ was also closely correlated with colistin activity, particularly for the low inoculum, in which no heteroresistant subpopulations were found. For the higher inoculum, including heteroresistant subpopulations, the fAUC/MIC was strongly correlated with colistin activity. However, a concentration-dependent killing was observed within $2 \mathrm{~h}$ with an $f C_{\text {max }} / \mathrm{MIC}>6$ possibly required to kill the more susceptible subpopulation without affecting the less susceptible subpopulation for which a high fAUC/MIC $>25$ was required in order to prevent regrowth. This may explain why CART analysis indicated both $f C_{\text {max }}$ /MIC and $F A U C / M I C$ as equally significant predictive PK/PD indices for killing the high inoculum. Colistin's concentration-dependent killing was previously described against $P$. aeruginosa and $A$. baumannii ${ }^{16,17}$ and an $f C_{\max } / \mathrm{MIC}>8-10$ was suggested for other concentration-dependent bactericidal drugs, ${ }^{18}$ although fAUC/MIC appears to be the pharmacodynamic driver. ${ }^{15,19}$ Similarly, single high doses of azithromycin, an fAUC/ MIC-dependent drug, were more efficacious than multidose regimens in preclinical infection models. ${ }^{20}$ In contrast to other concentration-dependent drugs, colistin exerts a strong initial concentration-dependent killing but no significant post-antibiotic effect at low, clinically relevant concentrations ( $1 \mathrm{~h}$ at the MIC and $2-3 \mathrm{~h}$ at $>16 \times \mathrm{MIC}) .{ }^{16,21}$ In addition, colistin has a relatively long half-life. However, given that a high $f C_{\text {max }} / \mathrm{MIC}$ cannot be obtained in patients' serum for isolates with an MIC $>0.25 \mathrm{mg} / \mathrm{L}$, the $\mathrm{fAUC/}$ MIC may be the determining PK/PD index in patients.

Based on a PK/PD target of 25 for the fAUC/MIC, Monte Carlo simulations showed a cumulative fraction of response for the WT distribution of $77 \%-83 \%$ for all three clinical dosing regimens. These rates are similar to the clinical cure rate of $82.1 \%$ found in patients with sepsis due to Gram-negative bacteria susceptible only to colistin and treated with 4.5 MU q12h. ${ }^{22}$ A similar cure rate of $83.3 \%$ was reported in another retrospective cohort study. ${ }^{23}$ However, lower clinical cure rates (57\%-73\%) have been reported in several retrospective studies in which lower colistin 
(a)

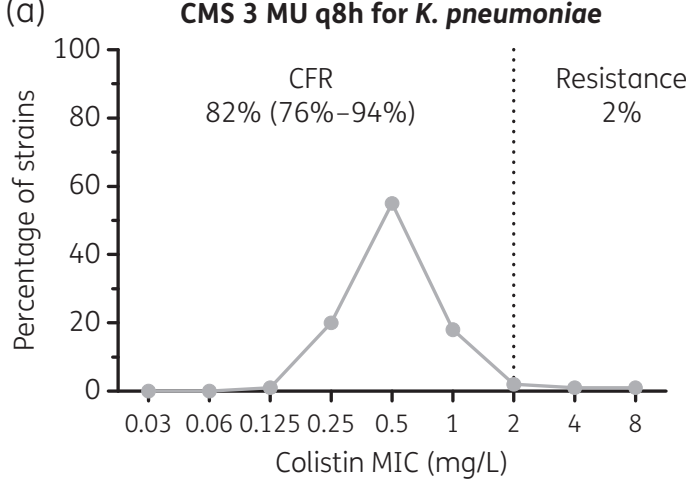

(c)

CMS 3 MU q8h for K. pneumoniae

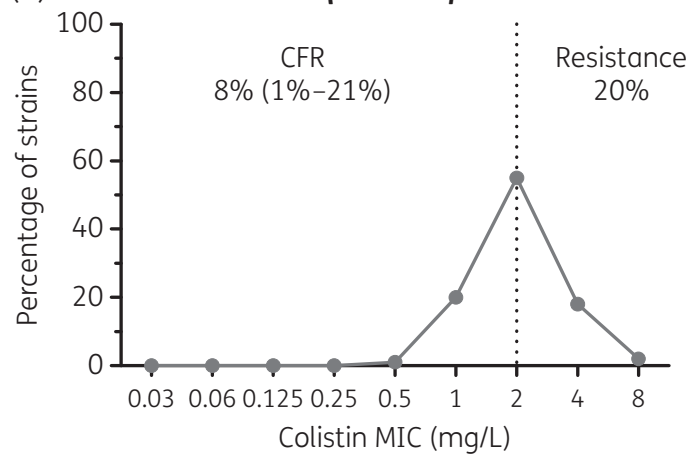

(b) $\quad$ CMS 3 MU q8h for K. pneumoniae

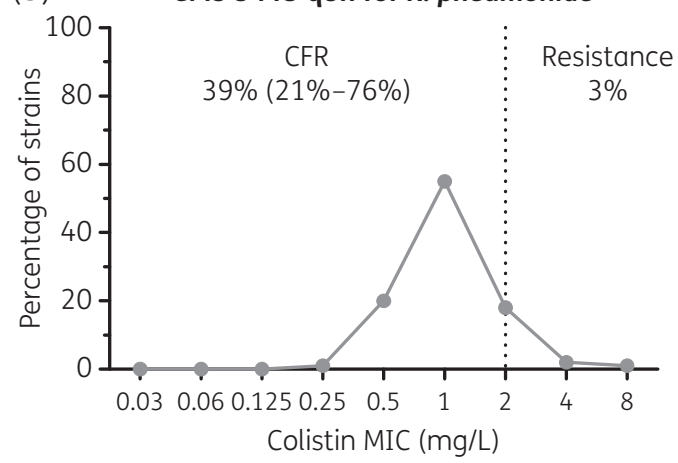

(d) CMS 3 MU q8h for K. pneumoniae

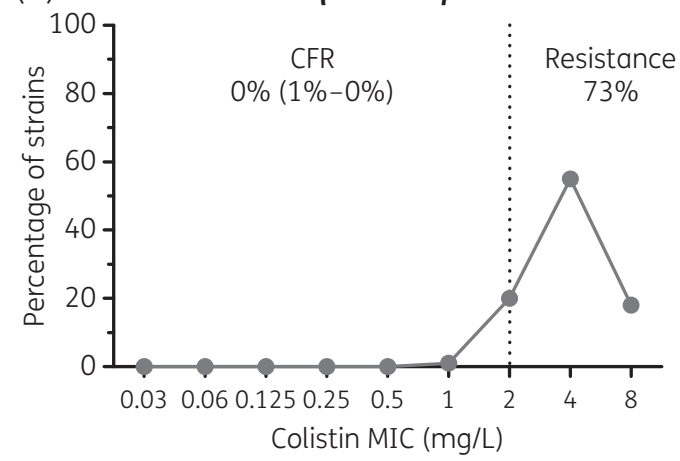

Figure 6. The cumulative fraction of response (CFR) defined as the population PTA for the $3 \mathrm{MU}$ q8h CMS dose (similar exposures were found for $4.5 \mathrm{MU}$ q12h and $9 \mathrm{MU}$ q24h) in ICU patients with normal renal function ${ }^{11}$ and a collection of isolates with modal MICs of 0.5 (EUCAST MIC distribution), 1, 2 and $4 \mathrm{mg} / \mathrm{L}$, with proportions of resistant isolates (MIC >2 mg/L, horizontal dotted line) of $2 \%, 3 \%, 20 \%$ and $73 \%$, respectively.

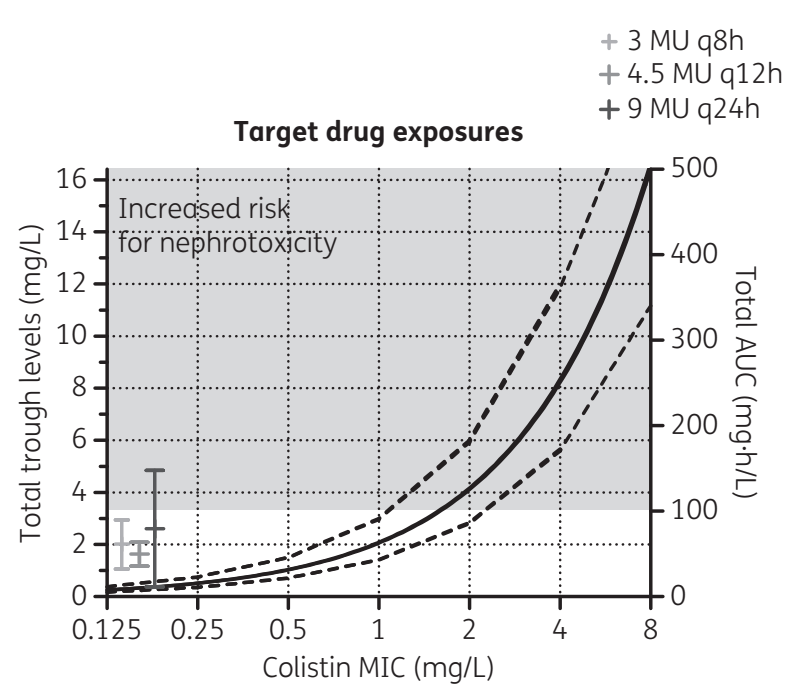

Figure 7. Target drug exposure (total trough levels on the left axis and total $\mathrm{AUC}_{0-24}$ on the right axis) required to attain the mean (95\% CI) PK/PD target fAUC/MIC of 25 (17-36) and the target tAUC of 62.5 (42.5-90) against $K$. pneumoniae isolates with different MICs. The shaded area indicates increased risk for nephrotoxicity, which is usually associated with trough levels $>3.33 \mathrm{mg} / \mathrm{L}$. The dotted lines represent the $95 \% \mathrm{CI}$ of the PK/PD target calculated from the in vitro model. Trough levels of the three clinical dosing regimens $9 \mathrm{MU}$ q24h, 4.5 MU q12h and $3 \mathrm{MU}$ q8h in ICU patients with normal renal function ${ }^{9}$ are shown next to the left-hand axis. methanesulphonate (CMS) doses (1-3 MU) were given to patients with ventilator-associated pneumonia. ${ }^{1}$ Such low daily doses would not produce drug exposures sufficient to attain the PK/PD target for isolates with an MIC of $0.5-1 \mathrm{mg} / \mathrm{L}$.

The lower success rates reported with colistin may be explained by inefficient drug exposure or infections by less susceptible isolates shifting out of the WT distribution. A recent surveillance multicentre study recording resistance rates for colistin of K. pneumoniae, E. coli, $P$. aeruginosa and $A$. baumanii isolates from ICU patients showed that although the resistance rate is low $(<10 \%)$, the median MIC is $1 \mathrm{mg} / \mathrm{L}, 24,25$ one 2 -fold dilution higher than the median MIC of the WT EUCAST distribution. As shown in the present study, this increase reduced the PTA rates significantly. In centres with such a shift in MICs or with higher resistance rates, ${ }^{26}$ clinical cure by colistin monotherapy is low and therefore alternative chemotherapeutic approaches are followed, e.g. combination therapy. ${ }^{27}$ Comparable high clinical cure rates were found for colistin monotherapy and combination therapy for isolates with an MIC of $\leq 0.5 \mathrm{mg} / \mathrm{L} .{ }^{28}$ Significantly, this MIC was found in the present study to be the highest MIC offering a reasonable PTA.

The optimal dose should be sufficient to result in a $t C_{\max } 7.5 \mathrm{mg} / \mathrm{L}$ $\left(f C_{\max }=3 \mathrm{mg} / \mathrm{L}\right)$ or tAUC of $31.25 \mathrm{mg} \cdot \mathrm{h} / \mathrm{L}(f A \cup C=12.5 \mathrm{mg} \cdot \mathrm{h} / \mathrm{L})$ for an isolate with an MIC of $0.5 \mathrm{mg} / \mathrm{L}$ (target $f C_{\max } / \mathrm{MIC}=6$ and $f A U C$ / $M I C=25$ ). Although high $t C_{\max } s$ up to $23 \mathrm{mg} / \mathrm{L}$ have been reported, ${ }^{29}$ few patients could attain such a high $t C_{\max }$ of $7.5 \mathrm{mg} / \mathrm{L}$ even with the highest dose of $9 \mathrm{MU} .{ }^{9}$ Thus, the tAUC of $31.25 \mathrm{mg} \cdot \mathrm{h} / \mathrm{L}$ is a more clinically feasible target for isolates with an MIC of up to 
$0.5 \mathrm{mg} / \mathrm{L}$. Assuming a half-life of $12 \mathrm{~h}$, a trough/MIC ratio of 2 should be targeted in order to obtain a tAUC of $31.25 \mathrm{mg} \cdot \mathrm{h} / \mathrm{L}$. Isolates with an MIC of up to $1 \mathrm{mg} / \mathrm{L}$, which represent $94 \%$ of the WT distribution, could be treated with a dosing regimen that produces a trough level of $2 \mathrm{mg} / \mathrm{L}$. Isolates with higher MICs would require higher trough levels $(>3.33 \mathrm{mg} / \mathrm{L})$, which are associated with an increased risk of nephrotoxicity. ${ }^{12}$ An average steady-state trough level of $>2 \mathrm{mg} / \mathrm{L}$ has been previously suggested as adequate for treatment of these infections. ${ }^{30}$ However, the large interindividual variability in $C_{\mathrm{ss}, \mathrm{avg}}$ (median 2.35, range $0.24-9.92 \mathrm{mg} / \mathrm{L}$ ) and in protein binding (mean \pm SD unbound fraction $0.49 \pm 0.11$ in critically ill patients) $^{31}$ indicates that most patients would attain the PK/PD target for isolates with an MIC of up to $0.5 \mathrm{mg} / \mathrm{L}$, whereas for isolates with an MIC of $1 \mathrm{mg} / \mathrm{L}$, TDM can optimize drug exposure by increasing efficacy and reducing toxicity. Alternatively, high infrequent doses (e.g. 12-18 MU q48h or q72h) may optimize both $C_{\max } / \mathrm{MIC}$ and AUC/MIC indices and reduce trough levels associated with nephrotoxicity, although toxicity studies are required to prove the safety of those dosing regimens.

Based on the PK/PD target of FAUC/MIC $>25$, a susceptibility PK/PD breakpoint of $\leq 0.5 \mathrm{mg} / \mathrm{L}$ was determined in the present study. This breakpoint is lower than the EUCAST clinical breakpoint and ECOFF value of $\leq 2 \mathrm{mg} / \mathrm{L}$. However, using the latter endpoint, $>98 \%$ of isolates would be deemed susceptible, whereas the success rate of colistin monotherapy hardly reaches $80 \%$ against colistin-susceptible isolates based on previous clinical studies. ${ }^{22}$ Of note, using the $0.5 \mathrm{mg} / \mathrm{L}$ susceptibility breakpoint, $78 \%$ would be susceptible. Furthermore, using the $2 \mathrm{mg} / \mathrm{L}$ breakpoint, no difference was found in mortality between carbapenem-resistant Enterobacteriaceae infections by colistinsusceptible and colistin-resistant isolates. ${ }^{32}$ More important is the finding that the clinical outcomes of colistin therapy in patients infected with susceptible (MIC $\leq 2 \mathrm{mg} / \mathrm{L}$ ) A. baumannii isolates but high colistin MICs (1-2 mg/L) were poorer than in patients infected with isolates with lower ( $<1 \mathrm{mg} / \mathrm{L}$ ) MICs (7 day outcome $38 \%$ versus $20.2 \%, P=0.025){ }^{33}$ Finally, it was suggested that current maintenance doses may not be effective against isolates with an MIC $>0.5 \mathrm{mg} / \mathrm{l} .{ }^{34}$ Thus, a revision of the current susceptibility breakpoint for colistin may be needed.

For isolates with an MIC $\leq 0.5 \mathrm{mg} / \mathrm{L}(<50 \%$ of the clinical isolates) monotherapy can attain the pharmacodynamic target, whereas for isolates with an MIC of $1 \mathrm{mg} / \mathrm{L}$, TDM can optimize drug exposure in order to attain the pharmacodynamic target. Target attainment rates may be higher for patients with reduced renal clearance though with increased risk for toxicity. For those patients, dose adjustments have been recommended based on creatinine clearance. ${ }^{33}$ Monotherapy will not be sufficient for isolates with higher MICs and combination therapy should be considered in order to decrease the pharmacodynamic target by synergistic interactions. For patients with augmented renal function, higher CMS doses (12-18 MU) may be used in order to achieve adequate levels of colistin. Those dose recommendations together with the nomogram of Figure 7 can be used in order to optimize CMS dosing regimens for patients with altered renal clearance. However, dose adjustments based on MICs require reliable and reproducible MIC testing assays and the in vitro PD target determined here needs to be comparable with the clinical PD target.

In conclusion, in vitro PK/PD modelling of colistin activity against K. pneumoniae showed that FAUC/MIC is the best predictor of colistin activity for clinically achievable concentrations, and $f C_{\max } /$ MIC the best determinant of colistin bactericidal activity. Colistin rapidly kills susceptible subpopulations in a concentrationdependent manner at $f C_{\max } / \mathrm{MIC}>6$, whereas for prolonged suppression of growth of resistant subpopulations an fAUC/MIC $>25$ is required. $A n f C$ max $/ \mathrm{MIC}>6$ can be attained with standard dosing regimens for isolates with an MIC $\leq 0.125 \mathrm{mg} / \mathrm{L}$, but very few of these exist. Because of the low $f C_{\max }$ achieved in human plasma, fAUC/MIC $>25$ is a more clinically feasible target. Target attainment rates drop rapidly for isolates with reduced susceptibility to colistin, necessitating TDM and the use of combination therapy.

\section{Funding}

This study was supported by the European Union 7th Framework Program (FP7) 'AIDA preserving old antibiotics for the future' (project number 278348).

\section{Transparency declarations}

None to declare.

\section{References}

1 Giamarellou H. Multidrug-resistant Gram-negative bacteria: how to treat and for how long. Int J Antimicrob Agents 2010; 36: S50-4.

2 Tzouvelekis LS, Markogiannakis A, Psichogiou M et al. Carbapenemases in Klebsiella pneumoniae and other Enterobacteriaceae: an evolving crisis of global dimensions. Clin Microbiol Rev 2012; 25: 682-707.

3 Bergen P, Landersdorfer C, Zhang J. Pharmacokinetics and pharmacodynamics of 'old' polymyxins: what is new? Diagn Microbiol Infect Dis 2012; 74: 213-23.

4 Clinical and Laboratory Standards Institute. Performance Standards for Antimicrobial Susceptibility Testing: Fifteenth Informational Supplement M100-S15. CLSI, Wayne, PA, USA, 2005.

5 Meletiadis J, Al-Saigh R, Velegraki A et al. Pharmacodynamic effects of simulated standard doses of antifungal drugs against Aspergillus species in a new in vitro pharmacokinetic/pharmacodynamic model. Antimicrob Agents Chemother 2012; 56: 403-10.

6 Tsala M, Vourli S, Kotsakis S et al. Pharmacokinetic-pharmacodynamic modeling of meropenem against VIM producing Klebsiella pneumoniae isolates: clinical implications. J Med Microbiol 2016; 65: 211-8.

7 Tam VH, Schilling AN, Neshat S et al. Optimization of meropenem minimum concentration/MIC ratio to suppress in vitro resistance of Pseudomonas aeruginosa. Antimicrob Agents Chemother 2005; 49: 4920-7.

8 Mohamed AF, Karaiskos I, Plachouras D et al. Application of a loading dose of colistin methanesulfonate in critically ill patients: population pharmacokinetics, protein binding, and prediction of bacterial kill. Antimicrob Agents Chemother 2012; 56: 4241-9.

9 Daikos GL, Skiada A, Pavleas J et al. Serum bactericidal activity of three different dosing regimens of colistin with implications for optimum clinical use. J Chemother 2010; 22: 175-8.

10 Mouton JW, Dudley MN, Cars O et al. Standardization of pharmacokinetic/pharmacodynamic (PK/PD) terminology for anti-infective drugs: an update. J Antimicrob Chemother 2005; 55: 601-7.

11 Plachouras D, Karvanen M, Friberg LE et al. Population pharmacokinetic analysis of colistin methanesulfonate and colistin after intravenous 
administration in critically ill patients with infections caused by gramnegative bacteria. Antimicrob Agents Chemother 2009; 53: 3430-6.

12 Sorlí L, Luque S, Grau S et al. Trough colistin plasma level is an independent risk factor for nephrotoxicity: a prospective observational cohort study. BMC Infect Dis 2013; 13: 380.

13 EUCAST. Rationale for the EUCAST Clinical Breakpoints, Version 1. C. Colistin. 2010; 1-10. http://www.eucast.org/fileadmin/src/media/PDFs/ EUCAST_files/Rationale_documents/Colistin_rationale_1.0.pdf.

14 Docobo-Pérez F, Nordmann P, Domínguez-Herrera J et al. Efficacies of colistin and tigecycline in mice with experimental pneumonia due to NDM-1producing strains of Klebsiella pneumoniae and Escherichia coli. Int $J$ Antimicrob Agents 2012; 39: 251-4.

15 Cheah S-E, Wang J, Nguyen VTT et al. New pharmacokinetic/pharmacodynamic studies of systemically administered colistin against Pseudomonas aeruginosa and Acinetobacter baumannii in mouse thigh and lung infection models: smaller response in lung infection. J Antimicrob Chemother 2015; 70 : 3291-7.

16 Li J, Turnidge J, Milne R et al. In vitro pharmacodynamic properties of colistin and colistin methanesulfonate against Pseudomonas aeruginosa isolates from patients with cystic fibrosis. Antimicrob Agents Chemother 2001; 45: 781-5.

17 Li J, Rayner CR, Nation RL et al. Heteroresistance to colistin in multidrugresistant Acinetobacter baumannii. Antimicrob Agents Chemother 2006; 50: 2946-50.

18 Levison ME. Pharmacodynamics of antimicrobial drugs. Infect Dis Clin North Am 2004; 18: 451-65.

19 Mouton JW, Jacobs N, Tiddens H et al. Pharmacodynamics of tobramycin in patients with cystic fibrosis. Diagn Microbiol Infect Dis 2005; 52: 123-7.

20 Girard D, Finegan SM, Dunne MW et al. Enhanced efficacy of single-dose versus multi-dose azithromycin regimens in preclinical infection models. J Antimicrob Chemother 2005; 56: 365-71.

21 Poudyal A, Howden BP, Bell JM et al. In vitro pharmacodynamics of colistin against multidrug-resistant Klebsiella pneumoniae. J Antimicrob Chemother 2008; 62: 1311-8.

22 Dalfino L, Puntillo F, Mosca A et al. High-dose, extended-interval colistin administration in critically ill patients: is this the right dosing strategy? A preliminary study. Clin Infect Dis 2012; 54: 1720-6.

23 Falagas ME, Rafailidis PI, Ioannidou E et al. Colistin therapy for microbiologically documented multidrug-resistant Gram-negative bacterial infections: a retrospective cohort study of 258 patients. Int J Antimicrob Agents 2010; 35: 194-9.

24 Jean SS, Lee WS, Yu KW et al. Rates of susceptibility of carbapenems, ceftobiprole, and colistin against clinically important bacteria collected from intensive care units in 2007: results from the Surveillance of Multicenter Antimicrobial Resistance in Taiwan (SMART). J Microbiol Immunol Infect 2014; 49: 969-76.

25 Sader HS, Huband MD, Castanheira M et al. Antimicrobial susceptibility of Pseudomonas aeruginosa: results from four years (2012-2015) of the International Network for Optimal Resistance Monitoring (INFORM) program in the United States. Antimicrob Agents Chemother 2017; 61: e02252-16.

26 Kontopidou F, Giamarellou H, Katerelos $P$ et al. Infections caused by carbapenem-resistant Klebsiella pneumoniae among patients in intensive care units in Greece: a multi-centre study on clinical outcome and therapeutic options. Clin Microbiol Infect 2014; 20: 0117-23.

27 Munoz-Price LS, Poirel L, Bonomo RA et al. Clinical epidemiology of the global expansion of Klebsiella pneumoniae carbapenemases. Lancet Infect Dis 2013; 13: 785-96.

28 Shah PG, Shah SR. Treatment and outcome of carbapenem-resistant Gram-negative bacilli blood-stream infections in a tertiary care hospital. J Assoc Physicians India 2015; 63: 14-8.

29 Karnik ND, Sridharan K, Jadhav SP et al. Pharmacokinetics of colistin in critically ill patients with multidrug-resistant Gram-negative bacilli infection. Eur J Clin Pharmacol 2013; 69: 1429-36.

30 Nation RL, Garonzik SM, Li J et al. Updated US and European dose recommendations for intravenous colistin: how do they perform? Clin Infect Dis 2016; 62: 552-8.

31 Nation RL, Garonzik S, Thamlikitkul V et al. Dosing guidance for intravenous colistin in critically-ill patients. Clin Infect Dis 2017; 64: 565-71.

32 de Maio Carrilho CMD, de Oliveira LM, Gaudereto J et al. A prospective study of treatment of carbapenem-resistant Enterobacteriaceae infections and risk factors associated with outcome. BMC Infect Dis 2016; 16: 629.

33 Choi IS, Lee YJ, Wi YM et al. Predictors of mortality in patients with extensively drug-resistant Acinetobacter baumannii pneumonia receiving colistin therapy. Int J Antimicrob Agents 2016; 48: 175-80.

34 Garonzik SM, Li J, Thamlikitkul V et al. Population pharmacokinetics of colistin methanesulfonate and formed colistin in critically ill patients from a multicenter study provide dosing suggestions for various categories of patients. Antimicrob Agents Chemother 2011; 55: 3284-94. 